\title{
Osteopathia striata in the mandible with cranial sclerosis: a case report and review of the literature
}

\author{
Rohan Jagtap ${ }^{1}$, Michelle Briner Garrido ${ }^{2}$, Matthew Hansen² \\ ${ }^{1}$ Division of Oral and Maxillofacial Radiology, Department of Care Planning and Restorative Sciences, University of Mississippi \\ Medical Center School of Dentistry, Jackson, MS, ${ }^{2}$ Division of Oral and Maxillofacial Radiology, Department of Oral and Maxillofacial \\ Diagnostic Sciences, University of Florida College of Dentistry, Gainesville, FL, USA
}

\begin{abstract}
J Korean Assoc Oral Maxillofac Surg 2021;47:141-144)
Osteopathia striata with cranial sclerosis (OS-CS) is a bone dysplasia characterized by a linear striated pattern of sclerosis, especially in the long bones, and cranial sclerosis. It has variable clinical findings but distinctive radiological findings. Multiple oral and dental findings have been associated with this disease and can be seen during dental and/or medical imaging of the head and neck. Dentists and clinicians must be familiar with these signs to differentiate them from pathosis or erroneous radiographs. In the following case, we present a patient with OS-CS that presented at The University of Florida College of Dentistry with multiple craniofacial manifestations of this syndrome that were seen on a panoramic radiograph, which is one of the most commonly requested radiographs by dentists.
\end{abstract}

Key words: Osteopathia striata with cranial sclerosis, Oral manifestation, Oral and maxillofacial radiology, Oral surgery

[paper submitted 2020. 2. 28 / revised 2020. 5. 8 / accepted 2020. 5. 8]

\section{Introduction}

Osteopathia striata (OS) is a bone dysplasia characterized by a linear striated pattern of sclerosis, especially in the long bones ${ }^{1}$. It was first described by Voorhoeve in 1924 and can occur in isolation or as part of a syndrome. Its association with cranial sclerosis (CS) was described first by Hurt in $1953^{2}$. Osteopathia striata with cranial sclerosis (OS-CS), also known as Horan-Beighton syndrome, is a genetic x-linked bone dysplasia caused by mutation in the AMER1 gene (also known as WTX or FAM123B) ${ }^{3,4}$. However, it also can occur by spontaneous mutation ${ }^{3-12}$. This condition typically affects females, with a 2.5:1 female predilection, in whom the clinical phenotype can be extremely variable, even within the

\section{Rohan Jagtap \\ Division of Oral and Maxillofacial Radiology, Department of Care Planning and Restorative Sciences, University of Mississippi Medical Center School of Dentistry, 2500 North State Street, D214-04, Jackson, MS 39216, USA \\ TEL: +1-601-984-6062 \\ E-mail:drrohanjagtap@gmail.com \\ ORCID: https://orcid.org/0000-0002-9115-7235}

(c) This is an open-access article distributed under the terms of the Creative Commons Attribution Non-Commercial License (http://creativecommons.org/ licenses/by-nc/4.0/), which permits unrestricted non-commercial use, distribution, and reproduction in any medium, provided the original work is properly cited. Copyright (C) 2021 The Korean Association of Oral and Maxillofacial Surgeons. All rights reserved. same family ${ }^{13}$. The male phenotype is highly variable, with fetal or neonatal lethality in most circumstances due to multiple congenital malformations ${ }^{6,8}$. The prevalence of OS-CS is approximately $0.1 / 1$ million people ${ }^{14}$, and the age of diagnosis varies from neonatal to the fifth decade ${ }^{5}$.

This condition has variable clinical findings but distinctive radiological findings ${ }^{8}$. Patients can be asymptomatic and accidentally diagnosed during radiographic examination or present with disabling physical anomalies sometimes leading to premature death ${ }^{5,15}$. The most characteristic radiographic feature of OS-CS are longitudinal striations of long bones and, in some cases, also of the pelvis and scapula, and sclerosis of the cranial vault and base $\mathrm{e}^{11,15}$. The striations are thin and well-defined and lie parallel to the long axis of an otherwise normal bone $e^{9,10}$. Histologically, these striations consist of dense bone with reduced marrow spaces ${ }^{16}$. These linear striations typically first appear between 5 months and 6 years of age $^{11}$.

Notwithstanding these pathognomonic signs of bone striations and base of the skull sclerosis, OS-CS exhibits great variability, ranging from mild skeletal manifestations to multisystem organ involvement due to variability of gene mutation or deletion ${ }^{3,15,17}$. Some common clinical findings are macrocephaly, headaches, facial paralysis due to narrowing 
of the foramina, cranial vault and base of the skull sclerosis, conductive hearing loss, sclerosis of the facial bones and mastoid area, hypertelorism, frontal bossing, broad and depressed nasal bridge, club feet, bifid spine, and mental retardation. Other reported but inconstant features include deformity of the sternum; cataracts; and cardiac, renal, and gastrointestinal defects $^{3,5,11-15,18}$. The CS is frequently disabling and can lead to conductive hearing loss due to pressure on the cranial nerves and/or fixation of the middle ear bones ${ }^{2,3,13,14}$. Nerve palsies are responsible for the headaches ${ }^{11}$.

Oral manifestations associated with this disease include cleft lip and/or palate, a high-arched palate, constricted maxilla, malocclusion, bifid uvula, microdontia, impacted teeth, and dense mandibular bone with visible striations. Deformed and short roots, ankylosis of the temporomandibular joints (TMJs), midface hypoplasia, and association with PierreRobin syndrome also have been reported ${ }^{11,13-16,18}$. These dental deformities can compromise the overall health of the patient. To the best of our knowledge, only one familial case has been published, where linear striations occurred in the mandible in a 38-year-old female and her 7-year-old son and 6-year-old daughter ${ }^{1}$.

The following report presents a case of OS-CS with multiple radiographic and dental findings.

\section{Case Report}

A 44-year-old male was referred to the University of Florida College of Dentistry for evaluation and treatment of the TMJs. His medical history was significant for OS-CS, cleft lip and palate, obstructive sleep apnea, conductive hearing loss, hypertension, bronchitis, asthma, radiculopathy, arthritis, diabetes, club feet, and chronic kidney disease.

A panoramic radiograph was performed to assess the general condition of the TMJs as the patient had previously undergone surgery for progressive bony growth and ankylosis of the joints. The radiographic assessment was limited due to severe sclerosis of the maxillofacial bones depicted as dense and diffuse lobulated radiopacities in the maxilla, mandible, TMJs, sphenoid, and temporal bones. The visualized cortical outline of the maxilla and mandible appeared dense, thick, and sclerotic. There were surgical microplates on the mandibular condyles and zygomatic arches as well as post-surgical discontinuity of the mandibular rami and subcondylar regions bilaterally.(Fig. 1)

Multiple dental findings were noted, including cleft lip and palate, oligodontia, retained deciduous teeth, bifid uvula, high arched palate, mandibular asymmetry, and TMJ ankyloses. These dental and maxillofacial findings are common clinical features, although not exclusive of OS-CS. However, with diagnosis of OS-CS, emphasis must be placed on the dental and maxillofacial examination since these finding are commonly seen in such patients.

Multiple surgeries were performed on multiple occasions on this patient to treat some of the previously mentioned defects including cleft lip and palate (1972), club feet (1973), ankyloses of the TMJs (1980) and hearing loss (1990). The surgery performed to treat hearing loss was a left ear tympanoplasty, and the patient currently is wearing hearing aids. Regarding this surgical intervention, there is literature mentioning that conductive hearing loss can be improved surgically $^{3,14}$. However, it has been unsuccessful in other patients ${ }^{3}$. At the present time, 40 years after the first TMJ surgery, our patient presented to the University of Florida College of Dentistry Clinic complaining of limited mouth opening and limited excursive movements of the mandible. A full TMJ replacement was recommended.

\section{Discussion}

OS-CS is a rare bone dysplasia with variable expression. It has very distinctive skeletal abnormalities including linear stri-
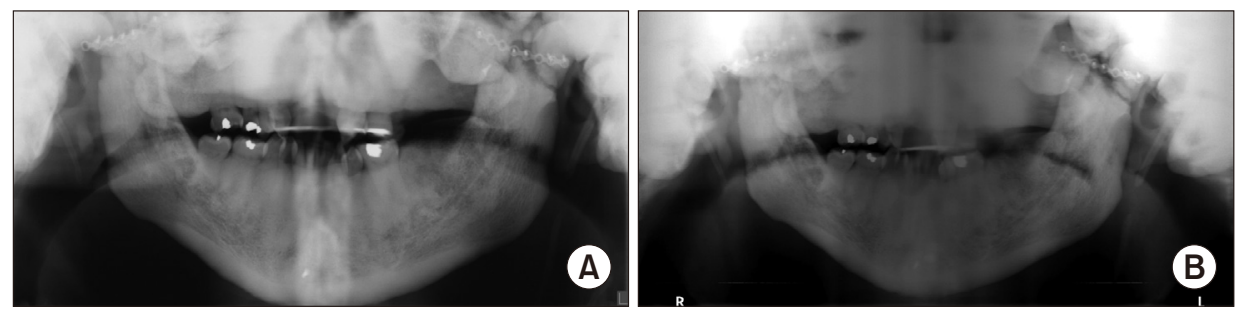

Fig. 1. A. In 2016, there is an increased bone mass of the cranium displayed as multiple lobulated, homogeneous radiopaque entities superimposing the maxilla, temporomandibular joints, sphenoid, and temporal bones. B. In 2017, the visualized cortical outline of the maxilla and mandible appeared dense, thickened and sclerotic.

Rohan Jagtap et al: Osteopathia striata in the mandible with cranial sclerosis: a case report and review of the literature. J Korean Assoc Oral Maxillofac Surg 2021 
ations of the long bones and $\mathrm{CS}^{19}$. It is important that diagnosis of OS is typically based on its radiographic appearance, and the striations are present in bones that can be otherwise normal.

The radiographic diagnosis of long bones can be made utilizing two- or three-dimensional images. Most of the cases in the literature have been incidental findings on two-dimensional images of the extremities since those are the most commonly requested by physicians. For evaluation of CS, the best diagnostic imaging system is computed tomography (CT) since it is a three-dimensional image that avoids superimposition of anatomy. Panoramic radiographs, widely used in dentistry, can show some disease signs but usually are not sufficient for diagnosis. Occasionally, CS will be ascertained from a panoramic due to superimpositions inherent to production of the radiograph. In the present case, we observed how cranial and mandibular sclerosis can be visualized on panoramic radiographs, but we were aware of the diagnosis of this patient before the radiograph was ordered, which aided in the interpretation.

There are multiple different bony dysplasias to be considered in differential diagnosis of OS-CS; however, none of them have the characteristic longitudinal striations of the long bones and calvarial thickening seen in OS-CS. These bony dysplasias include osteopetrosis, Paget disease, osteopoikilosis, hyperostosis corticalis generalisata, dyschodroplasia, pycnodysostosis, sclerosteosis, craniometaphyseal dysplasia, fibrous dysplasia, Camurati-Engelmann disease, frontometaphyseal dysplasia, dysosteosclerosis, and hyperostosis cranialis interna ${ }^{2,14,20-22}$. It is not always possible to distinguish between the various kinds of sclerotic bone dysplasias on the basis of cranial involvement in CT scans. As such, the majority require a radiologic examination of the whole skeleton or genetic testing. These syndromes, including OS-CS, should be considered when CS is evident on panoramic radiograph. Diagnosis is often made by a physician after a complete medical examination. Occasionally osteopatria striata can coexist with another disorder, known as overlap syndrome ${ }^{2}$. Information about OS-CS is incomplete, and the prognosis and relationship with other sclerosing bone dysplasias remain indefinite ${ }^{1}$.

Our patient did exhibit many of the reported dental features of the disease including cleft lip and palate, club feet, high palatal vault, oligodontia, bifid uvula, conductive hearing loss, and ankyloses of the TMJs. In terms of treatment options for these abnormalities, surgical intervention is the most common approach.

Cranial nerve deficiencies have occurred due to encroachment of the neural foramina or canals by sclerosis with involvement of the optic, trigeminal, and facial nerves report$\mathrm{ed}^{14}$. In our patient, mild right temporal and mandibular marginal branch weakness was noted; otherwise, cranial nerves five and seven were functioning as expected. Progression of the CS during adulthood has not been demonstrated in the scientific literature, and there is no proven medical treatment for it ${ }^{12}$.

Treatment for high palate and constricted maxilla includes distraction osteogenesis with a maxillary expander and orthodontic treatment; however, such interventions are very difficult given the density of the moving bone and the challenge of realignment of dentition. The density of the bone requires much time for adequate mobilization of the maxillary segment ${ }^{13,16}$.

All dental abnormalities of this patient were treated regardless of diagnosis of OS-CS, including cleft lip and palate repair, multiple restorations, extractions of damaged teeth, and TMJ surgery. Other treatments were suggested to the patient such as implant placement and orthodontic treatment. However, given the cost and difficulty associated with these treatments, the patient declined, suggesting a poor prognosis regarding dental rehabilitation.

As the prevalence of OS-CS is low and not all previously described dental anomalies are present in all cases, generalized dental treatment recommendations are limited and not often described. Furthermore, each case found in the literature was individually evaluated and, as in our case, treated as a regular dento-maxillofacial patient regardless of its syndromic association ${ }^{16}$.

In conclusion, OS-CS is a complex bone dysplasia with multiple clinical and dental manifestations. Diagnosis is generally based upon characteristic radiographic findings and clinical examination. Oral and maxillofacial radiologists, surgeons, and dentists should know the multiple dental features associated with this syndrome, and special emphasis must be applied to detect them during clinical and radiographic evaluation. Additionally, non-diagnosed patients can show striation of the bones in the mandible. If that is the case, this radiographic sign should call the attention of the oral radiologist, oral surgeon, or dentist, who should refer the patient to the physician for a complete examination.

Sclerosis of the skull base will impact visualization of the maxillofacial skeleton on panoramic radiographs given its increased radiopacity and superimposition over other anatomy. It is important to understand how sclerosis of the base of the skull will look like on a panoramic radiograph so we do not confuse it with technical problems during image acquisition. 
Considering the limitations of panoramic radiographs, conebeam CT might be the imaging modality of choice for such individuals.

\section{ORCID}

Rohan Jagtap, https://orcid.org/0000-0002-9115-7235

Michelle Briner Garrido, https://orcid.org/0000-0002-07379848

Matthew Hansen, https://orcid.org/0000-0001-6096-4264

\section{Authors' Contributions}

R.J. wrote the manuscript. M.B.G. participated in the study design and coordination. M.H. identified pathology and corrected the manuscript. All authors read and approved the final manuscript.

\section{Acknowledgements}

We thank the University of Florida Department of Oral and Maxillofacial Surgery.

\section{Conflict of Interest}

No potential conflict of interest relevant to this article was reported.

\section{References}

1. Nakamura T, Yokomizo Y, Kanda S, Harada T, Naruse T. Osteopathia striata with cranial sclerosis affecting three family members. Skeletal Radiol 1985;14:267-9. https://doi.org/10.1007/BF00352617

2. Magliulo G, Parrotto D, Zicari AM, Zappala D, Lo Mele L, Primicerio $\mathrm{P}$, et al. Osteopathia striata-cranial sclerosis: otorhinolaryngologic clinical presentation and radiologic findings. Am J Otolaryngol 2007;28:59-63. https://doi.org/10.1016/j.amjoto.2006.05.003

3. Lüerssen K, Ptok M. Osteopathia striata with cranial sclerosis and hearing loss. Eur Arch Otorhinolaryngol 2006;263:123-6. https:// doi.org/10.1007/s00405-005-0972-8

4. Vasiljevic A, Azzi C, Lacalm A, Combourieu D, CollardeauFrachon S, Dijoud F, et al. Prenatal diagnosis of osteopathia striata with cranial sclerosis. Prenat Diagn 2015;35:302-4. https://doi. org/10.1002/pd.4513

5. Zicari AM, Tarani L, Perotti D, Papetti L, Nicita F, Liberati N, et al. WTX R353X mutation in a family with osteopathia striata and cranial sclerosis (OS-CS): case report and literature review of the disease clinical, genetic and radiological features. Ital J Pediatr 2012;38:27. https://doi.org/10.1186/1824-7288-38-27

6. Hague J, Delon I, Brugger K, Martin H, Sparnon L, Simonic I, et al. Male child with somatic mosaic osteopathia striata with cranial sclerosis caused by a novel pathogenic AMER1 frameshift mutation. Am J Med Genet A 2017;173:1931-5. https://doi.org/10.1002/ ajmg.a.38261

7. Kondoh T, Yoshinaga M, Matsumoto T, Takayanagi T, Uetani M, Kubota T, et al. Severe cervical kyphosis in osteopathia striata with cranial sclerosis: case report. Pediatr Radiol 2001;31:659-62. https://doi.org/10.1007/s002470100486

8. Fradin M, Collet C, Ract I, Odent S, Guggenbuhl P. First case of osteopathia striata with cranial sclerosis in an adult male with Klinefelter syndrome. Joint Bone Spine 2017;84:87-90. https://doi. org/10.1016/j.jbspin.2016.04.012

9. Enomoto Y, Tsurusaki Y, Harada N, Aida N, Kurosawa K. Novel AMER1 frameshift mutation in a girl with osteopathia striata with cranial sclerosis. Congenit Anom 2018;58:145-6. https://doi. org $/ 10.1111 /$ cga. 12258

10. O’Byrne JJ, Phelan E, Steenackers E, van Hul W, Reardon W. Germline mosaicism in osteopathia striata with cranial sclerosis-recurrence in siblings. Clin Dysmorphol 2016;25:45-9. https://doi. org/10.1097/MCD.0000000000000116

11. Ward LM, Rauch F, Travers R, Roy M, Montes J, Chabot G, et al. Osteopathia striata with cranial sclerosis: clinical, radiological, and bone histological findings in an adolescent girl. Am J Med Genet A 2004;129A:8-12. https://doi.org/10.1002/ajmg.a.30107

12. Lazar CM, Braunstein EM, Econs MJ. Clinical vignette: osteopathia striata with cranial sclerosis. J Bone Miner Res 1999;14:152-3. https://doi.org/10.1359/jbmr.1999.14.1.152

13. Koudstaal MJ, Wolvius EB, Ongkosuwito EM, van der Wal KG. Surgically assisted rapid maxillary expansion in two cases of osteopathia striata with cranial sclerosis. Cleft Palate Craniofac J 2008;45:337-42. https://doi.org/10.1597/07-016

14. Berenholz L, Lippy W, Harrell M. Conductive hearing loss in osteopathia striata-cranial sclerosis. Otolaryngol Head Neck Surg 2002;127:124-6. https://doi.org/10.1067/mhn.2002.124852

15. Ng DW. A case study of a preadolescent with osteopathia striata with cranial sclerosis. J Pediatr Health Care 2017;31:511-6. https:// doi.org/10.1016/j.pedhc.2017.01.003

16. Goodman JR, Robertson CU. Osteopathia striata--a case report. Int J Paediatr Dent 1993;3:151-6. https://doi.org/10.1111/j.1365263x.1993.tb00072.x

17. Savarirayan R, Nance J, Morris L, Haan E, Couper R. Osteopathia striata with cranial sclerosis: highly variable phenotypic expression within a family. Clin Genet 1997;52:199-205. https://doi. org/10.1111/j.1399-0004.1997.tb02547.x

18. Daley TD, Wysocki GP, Bohay RN. Osteopathia striata, short stature, cataracts, and microdontia: a new syndrome? A case report. Oral Surg Oral Med Oral Pathol Oral Radiol Endod 1996;81:35660. https://doi.org/10.1016/s1079-2104(96)80337-4

19. Gay BB Jr, Elsas LJ, Wyly JB, Pasquali M. Osteopathia striata with cranial sclerosis. Pediatr Radiol 1994;24:56-60. https://doi. org/10.1007/BF02017665

20. Jagtap R, Alansari R, Ruprecht A, Kashtwari D. Trichodentoosseous syndrome: a case report and review of literature. BJR Case Rep 2019;5:20190039. https://doi.org/10.1259/bjrcr.20190039

21. Tomita Y, Chong PF, Yamamoto T, Akamine S, Imaizumi T, Kira R. Sequential radiologic findings in osteopathia striata with cranial sclerosis. Diagn Interv Imaging 2019;100:529-31. https://doi. org/10.1016/j.diii.2019.04.001

22. Ihde LL, Forrester DM, Gottsegen CJ, Masih S, Patel DB, Vachon LA, et al. Sclerosing bone dysplasias: review and differentiation from other causes of osteosclerosis. Radiographics 2011;31:186582. https://doi.org/10.1148/rg.317115093

How to cite this article: Jagtap R, Briner Garrido M, Hansen M. Osteopathia striata in the mandible with cranial sclerosis: a case report and review of the literature. J Korean Assoc Oral Maxillofac Surg 2021;47:141-144. https://doi.org/10.5125/jkaoms.2021.47.2.141 